\title{
Microhaemangiomas of the iris with spontaneous hyphaema and acute glaucoma
}

\author{
HENRY D. PERRY,* FREDERIC J. MALLEN, AND WALTER SUSSMAN \\ From the Department of Surgery, Division of Ophthalmology, Nassau County Medical Center, \\ East Meadow, New York
}

SUMMARY A case of bilateral microhaemangiomas of the pupillary borders in a 69-year-old woman is described. When first seen she presented with acute angle closure glaucoma and spontaneous hyphaema. Photographs and iris fluorescein angiography are presented and microhaemangiomas are discussed.

\section{Case report}

A 69-year-old White woman was referred to the Nassau County Medical Center in July 1975 with a diagnosis of acute glaucoma and spontaneous hyphaema. On the evening before admission she noted decreased vision in the left eye. On awakening the following morning vision had worsened and the eye had become painful. Two hours later her referring ophthalmologist noted a corrected visual acuity of 20/400 in the left eye, a hazy cornea, and a middilated pupil. Immediate treatment included intravenous acetazolamide $500 \mathrm{mg}$ and $2 \%$ pilocarpine drops every five minutes for 20 minutes. The ocular history was normal and there was no history of trauma. On examination the physical findings were normal except for mild emphysema.

On admission the corrected visual acuity of the right eye was 20/20-1 and the left eye was 20/200. The intraocular pressure by applanation was 22 $\mathrm{mmHg}$ in the right eye compared with $50 \mathrm{mmHg}$ in the left eye. The right eye was normal except for microhaemangiomas at the pupillary border at the 9 o'clock position. The left cornea had microcystic epithelial oedema, and the left pupil measured $3 \mathrm{~mm}$ and was poorly reactive to light. The anterior chamber was shallow with a small hyphaema inferiorly and a bleeding site from a prominent cluster of fine vessels at the 2 o'clock position on the

\footnotetext{
*Present affiliation: Postdoctorial Fellow, Eye Research Institute of Retina Foundation, 20 Staniford Street, Boston, Massachusetts 02114, USA
}

Supported in part by a Fight for Sight Postdoctoral Research Fellowship No. F-300, Fight for Sight, Inc., New York City

Address for reprints: Henry D. Perry, MD, 112 Main Street, East Rockaway, New York 11518, USA pupillary border. These clusters of small vessels were coiled and appeared very thin-walled. These vascular tufts were more prominent in the left eye. Both irides were pale blue with a well defined collarette, and neither showed evidence of rubeosis or persistent pupillary membrane. The lens and posterior segment were unremarkable. Gonioscopy, performed later, showed a closed angle superiorly and an open but narrow angle inferiorly. After 20 minutes the bleeding stopped.

That evening therapy with pilocarpine $2 \%$, oral acetazolamide $250 \mathrm{mg}$ four times a day, and oral glycerol resulted in clearing the corneal oedema and lowering the ocular tension to $20.6 \mathrm{mmHg}$. The next day the visual acuity of the left eye was $20 / 25$, the cornea and anterior chamber were clear, and the intraocular pressure with applanation was 16 $\mathrm{mmHg}$. The patient was treated with bed rest for an additional four days, during which time the anterior chamber remained clear and the intraocular pressure remained within normal limits despite discontinuation of all antiglaucoma medications.

During the follow-up period there was no recurrence of the hyphaema. A dark-room provocative test showed an increase of $8 \mathrm{mmHg}$ in both eyes and was followed by uneventful bilateral peripheral iridectomies. Postoperative photographs were taken, and fluorescein angiography of the irides was performed (Figs. 1 and 2). Examination of family members contributed no relevant information.

\section{Discussion}

Microhaemangiomas, or vascular tufts, are small lesions consisting of minute, tightly-coiled blood vessels with thin, delicate, capillary-like walls that form at the ruff of the pupillary border (Fechner, 


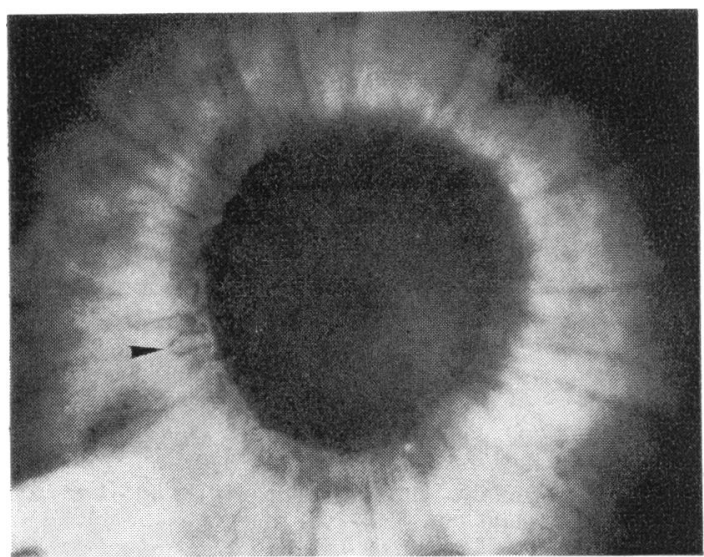

Fig. 1 Slit-lamp photograph of right eye; microhaemangioma at 9 o'clock meridian (arrow)

1958; Cobb, 1968; Rosen and Lyons, 1969; Cobb et al., 1970; Sellman, 1972; Magauran, 1973; Israel and Lorenzetti, 1974; Savir and Manor, 1975). They may be isolated or may arrange themselves in small clusters that have no tendency toward network formation. Occasionally, the normal pigmentation of the pupillary ruff is altered near the microhaemangiomas (Cobb, 1968). These vascular tufts are uncommon, with approximately 100 cases previously reported (Fechner, 1958; Cobb, 1968; Rosen and Lyons, 1969; Cobb et al., 1970; Sellman, 1972; Magauran, 1973; Israel and Lorenzetti, 1974; Savir and Manor, 1975). In our clinical experience and in Sellman's (1972) microhaemangiomas occur in approximately one in every 2000 patients. The majority are bilateral, with only 13 unilateral cases reported (Fechner, 1958; Magauran, 1973; Savir and Manor, 1975). A slight male predominance has been noted, and the onset is usually in the sixth and seventh decades, except in myotonic dystrophy; even when microhaemangiomas accompany this disease, the cases tend to occur in the fourth and fifth decades (Cobb, et al., 1970). Among other associated conditions are respiratory disease and possibly diabetes mellitus.
The diagnosis of microangiomas is made from the characteristic morphological appearance of these lesions as seen through the slit-lamp biomicroscope. They are easily missed and are difficult to photograph because of their small size. Fluorescein angiography, however, enables demonstration of a larger number of these lesions than are apparent on slitlamp examination (Cobb, 1968; Rosen and Lyons, 1969). Although the aetiology remains unknown, it would appear that these lesions are developmental in view of their distinctly higher incidence among the elderly (Cobb, 1968; Cobb, et al., 1970).

In no previous report did a patient present with a spontaneous hyphaema and acute glaucoma (Table 1). Magauran (1973) reported a case in which secondary glaucoma developed in association with a recurrent hyphaema several years after the first episode. The size of the hyphaema was not stated. However, despite resolution of the hyphaema and normal findings on ocular examination, including gonioscopy, administration of pilocarpine $4 \%$

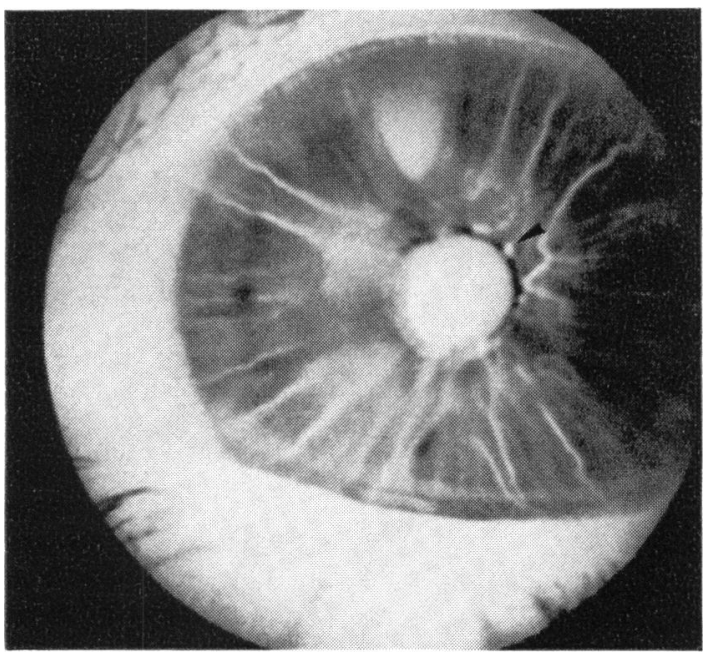

Fig. 2 Fluorescein angiography of the left eye depicting microhaemangiomas as dots of hyperfluorescence at the pupillary border. Arrow indicates site that previously bled

Table 1 Summary of cases of microhaemangioma with spontaneous hyphaema

\begin{tabular}{llllll}
\hline Reference & & Age and sex & Bilateral & Associated findings & Fluorescein angiography \\
\hline Fechner & $(1958)$ & $42 \mathrm{~F}$ & No & None & No \\
Magauran & $(1973)$ & $62 \mathrm{M}$ & Yes & Not stated & Yes \\
Magauran & $(1973)$ & $61 \mathrm{~F}$ & No & Not stated & Yes \\
Magauran & $(1973)$ & $69 \mathrm{~F}$ & Yes & Not stated & Yes \\
Rosen et al. & $(1969)$ & $73 \mathrm{M}$ & Yes & Chronic bronchitis, hypertension \\
Israel et al. & $(1974)$ & $45 \mathrm{~F}$ & Yes & Mild osteoarthritis & Yes \\
Sellman & $(1972)$ & $76 \mathrm{~F}$ & Yes & None & No \\
Sellman & $(1972)$ & $47 \mathrm{~F}$ & No & Congestive heart failure & Yes \\
Savir et al. & $(1975)$ & & & & \\
\hline
\end{tabular}


four times a day was necessary to maintain the ocular tension within normal limits. Savir and Manor (1975) reported a case in which the ocular tension increased from 14 to $22 \mathrm{mmHg}$ during an episode of hyphaema secondary to bleeding from a microhaemangioma. Gonioscopic examination disclosed a wide open angle, and the water provocative test was negative. In our case the patient was predisposed to acute glaucoma, and the attack was precipitated by the occurrence of a spontaneous hyphaema. After treatment the tension returned to normal without any medications. It appears likely that the glaucoma or increased intraocular pressure that may occur in a patient with microhaemangiomas and spontaneous hyphaema is either a direct result of the hyphaema or the presence of undiagnosed glaucoma. The presence of microhaemangiomas alone does not seem to be related to an increased incidence of glaucoma.

Owing to the scarcity of reports of iris microhaemangiomas association with spontaneous hyphaema and glaucoma cannot as yet be determined, and only a generalised clinical picture can be given. Perhaps if more attention is directed towards studying these vascular abnormalities questions on their true in- cidence, aetiology, associated conditions, and prognosis can be fully answered.

We thank Dr. Jack S. Nauheim for help in reviewing the manuscript and Dr. R. C. McCann for referring the patient. Illustrations were prepared by the Department of Medical Illustrations, Nassau County Medical Center. Editorial assistance was provided by S. Flavia Blackwell, Eye Research Institute of Retina Foundation.

\section{References}

Cobb, B. (1968). Transactions of the Ophthalmological Societies of the United Kingdom, 88, 211.

Cobb, B., Shilling, J. S., and Chisolm, I. H. (1970). American Journal of Ophthalmology, 69, 573.

Fechner, P. V. (1958). British Journal of Ophthalmology, 42, 311.

Israel, M. P., and Lorenzetti, D. W. C. (1974). Canadian Journal of Ophthalmology, 9, 138.

Magauran, D. M. (1973). British Journal of Ophthalmology, 57, 945.

Rosen, E., and Lyons, D. (1969). American Journal of Ophthalmology, 67, 846.

Savir, H., and Manor, R. S. (1975). Archives of Ophthalmology, 93, 1056.

Sellman, A. (1972). Acta Ophthalmologica, 50, 58. 